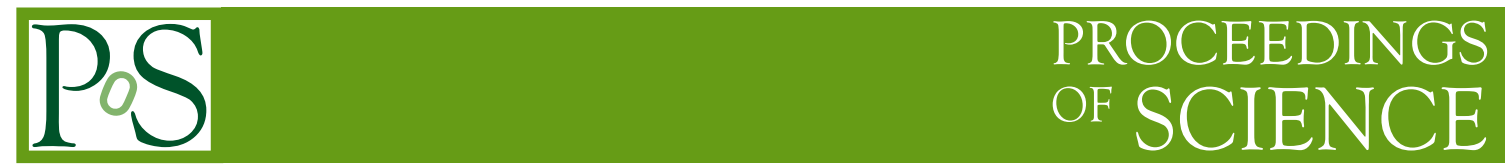

\title{
The SuperKEKB accelerator status
}

\author{
Masako Iwasaki*† \\ High Energy Accelerator Research Organization (KEK), Tsukuba, Ibaraki 305-0801 Japan \\ E-mail: masako.iwasaki@kek.jp
}

For the next generation B-factory experiment in Japan, SuperKEKB, the high luminosity $e^{+} e^{-}$ asymmetric collider at the B mesons CM energy, is planed as an upgrade of the current KEKB. It is designed to achieve a luminosity of $8 \times 10^{35} / \mathrm{cm}^{2} / \mathrm{s}$, 40 times higher than the highest luminosity record at KEKB. A summary of the current machine deign and R\&D status is presented.

35th International Conference of High Energy Physics

July 22-28, 2010

Paris, France

\footnotetext{
*Speaker.

${ }^{\dagger}$ On behalf of the SuperKEKB accelerator group.
} 


\section{Introduction}

SuperKEKB is the upgrade plan of the current B-factory experiment with the KEKB $e^{+} e^{-}$ accelerator at KEK[1]. Its luminosity is designed to be $8 \times 10^{35} / \mathrm{cm}^{2} / \mathrm{s}$ (40 times higher than KEKB) and the integrated luminosity is expected to be $50 \mathrm{ab}^{-1}$.

To achieve the 40 times higher luminosity, SuperKEKB employs the "nano-beam" scheme, which was first proposed for the Super B factory in Italy[2]. Here beams are squeezed to the nanometer scale and the beam crossing angle is enlarged so that beam bunches only intersect at the highly focused region.

Table 1 shows the KEKB and SuperKEKB machine parameters. In SuperKEKB, smaller beam size and higher beam current make the higher luminosity, and the larger crossing angle is adopted for the nano-beam scheme. Beam energies are changed from 3.5 and $8 \mathrm{GeV}$ to 4 and $7 \mathrm{GeV}$ to achieve the longer Touschek lifetime and mitigate the effect of intra-beam scattering in the $e^{+}$low energy ring beam (LER), and also helps lower emittance in the $e^{-}$high energy ring beam (HER).

\begin{tabular}{lccccc}
\hline \hline & \multicolumn{2}{c}{ KEKB } & \multicolumn{2}{c}{ SuperKEKB } & \\
& LER & HER & LER & HER & \\
\hline Energy & 3.5 & 8.0 & 4.0 & 7.0 & $\mathrm{GeV}$ \\
Half crossing angle & \multicolumn{2}{c}{11} & \multicolumn{2}{c}{41.5} & $\mathrm{mrad}$ \\
Horizontal emittance & 18 & 24 & 3.2 & 5.3 & $\mathrm{~nm}$ \\
Emittance ratio & 0.88 & 0.66 & 0.27 & 0.24 & $\%$ \\
Beta functions at IP & $1200(\mathrm{x}) / 5.9(\mathrm{y})$ & $32 / 0.27$ & $25 / 0.31$ & $\mathrm{~mm}$ \\
Beam currents & 1.64 & 1.19 & 3.6 & 2.6 & $\mathrm{~A}$ \\
Beam-beam parameter & 0.129 & 0.090 & 0.09 & 0.081 & \\
Luminosity & \multicolumn{2}{c}{$2.1 \times 10^{34}$} & \multicolumn{2}{c}{$8 \times 10^{35}$} & $\mathrm{~cm}^{-2} \mathrm{~s}^{-1}$ \\
\hline
\end{tabular}

Table 1: KEKB and SuperKEKB machine parameters.

For the SuperKEKB construction, the major items to be upgraded are:

- New antechamber beam pipes.

Developing the mitigation techniques to suppress the electron cloud.

- New interaction region (IR) design.

Construct the new superconducting final focusing magnets.

Optimization of the IR compensation solenoid.

Having the local chromaticity correction sections for both rings.

- New low emittance beam optics for both $e^{+} e^{-}$rings.

Replace dipoles, and change the wiggler layout for positron ring.

Install wiggler magnets to electron ring.

- New low emittance beam injections.

Positron damping ring and new positron target.

New RF gun for electrons with reduced emittance. 
- Higher beam currents.

Rearrangement of existing ARES cavities with additional power sources.

- Precise beam diagnostics and tunings.

New power supplies for more precise magnet setting.

\section{Vacuum System}

In the SuperKEKB vacuum system, it is important to suppress the heating of vacuum components by the synchrotron radiation (SR) and the higher-order mode (HOM) excitation. In addition, the electron cloud effect in LER and the fast ion effect in HER become severe.

The beam pipes for LER, the HER Tsukuba straight section, and the HER wiggler section are replaced with new beam pipes. A schematic view of the beam pipe with antechambers for LER is shown in Fig. 1. Beam goes through a beam channel, and SR goes into the antechamber, which is outside of the ring (SR channel). Due to this antechamber structure, the SR power density can be reduced. In addition, the antechamber scheme makes a smaller beam impedance. It also, in combination with TiN coating, reduces the electron cloud instability in LER[3].

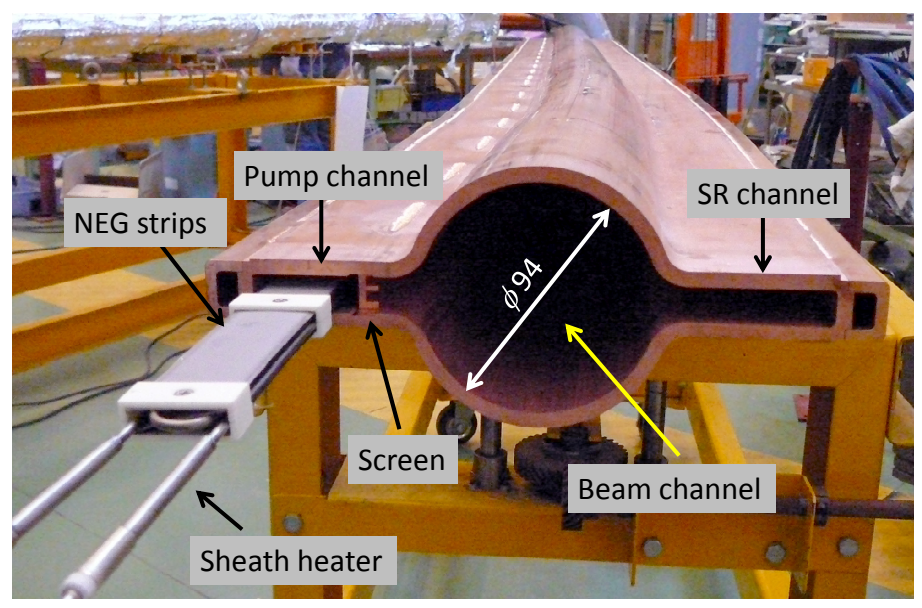

Figure 1: Beam pipe with antechambers for LER.

\section{IR Design}

Fig. 2 shows the schematic layout of the SuperKEKB IR, consisting with the 8 superconducting quadrupole magnets. The solenoid field of the Belle-II detector is compensated with compensation solenoids on each side of the interaction point (IP). The superconducting quadrupole and solenoid magnets on each side of the IP are contained in a common cryostat. These magnets surround a warm bore vacuum chamber connected to the cryostats via bellows.

The leakage fields of the superconducting magnets are canceled by correction windings on the opposite beam pipes. In recent studies of beam dynamics, it was found that the fringe fields of the compensation solenoids increase the vertical beam emittance. To reduce this effect, the 


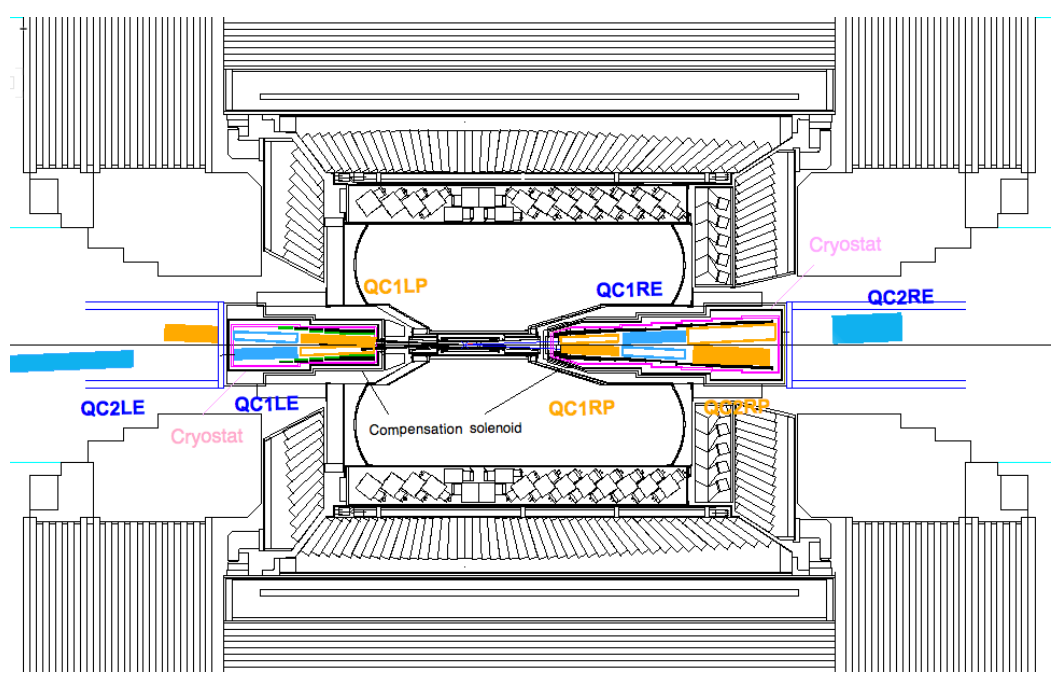

Figure 2: Schematic layout of the magnets and beam line near the interaction point.

compensation solenoids are segmented into small coil pieces to produce a slow gradient of the solenoid fringe field along the Belle-II axis. It is also important to make the total integrated solenoid field within the IR region to be 0 , to reduce the effect.

The new IP beam pipe design is also developed for SuperKEKB. Since there are two separate final focusing magnets in both $e^{+} e^{-}$beams, the IP beam pipe has the crotched structures on both left and right sides. We design the IP beam pipe radius of $1 \mathrm{~cm}$, the same radius as the final focusing magnet beam pipe, to avoid the resonant cavity structure. To prevent the direct SR hit to the BelleII detector, we put $4.5 \mathrm{~mm}$ radius mask on the IP beam pipe. For the precise beam orbit handling, beam position monitors are mounted near the IP.

\section{Ring beam optics}

We construct the beam optics design to achieve low emittance with minimal hardware change. For LER, we replace about 100 of $0.89 \mathrm{~m}$ length dipole magnets, which are used for KEKB, with $4 \mathrm{~m}$ length ones in SuperKEKB. In addition, the wiggler period will be shortened. In HER, we install wiggler magnets which are used for KEKB LER to reduce the emittance.

\section{Damping Ring}

The injected beam must have very low emittance to fit within the dynamic aperture of the main rings. We decide to construct a damping ring for positrons and a low emittance RF-gun for electrons. The damping ring has a FODO cell with alternating dipole magnets, where one of the two dipoles in each cell is reversed [4]. The damping ring parameters are summarized in Table 2. 


\begin{tabular}{lcc}
\hline \hline Energy & 1.1 & $\mathrm{GeV}$ \\
Circumference & 135 & $\mathrm{~m}$ \\
Number of trains, Number of bunches/train & 2,2 & \\
Maximum Stored current & 70.8 & $\mathrm{~mA}$ \\
Horizontal damping time & 11 & $\mathrm{~ms}$ \\
Injected-beam emittance & 1700 & $\mathrm{~nm}$ \\
Emittance at extraction & $42.5(\mathrm{H}) / 2.07(\mathrm{~V})$ & $\mathrm{nm}$ \\
Momentum compaction factor & 0.0141 & \\
Energy spread & 0.055 & $\%$ \\
\hline \hline
\end{tabular}

Table 2: Damping Ring Parameters.

\section{Summary}

On June 30th 2010, the KEKB operation was stopped to start the SuperKEKB upgrade. Currently the SuperKEKB construction is ongoing. Fig. 3 shows the luminosity projection for SuperKEKB. It is planed to start the commissioning in late FY2014, and we expect the design luminosity is achieved in around 2019. With this assumption, our integrated luminosity will reach 50 $\mathrm{ab}^{-1}$ in 2021.

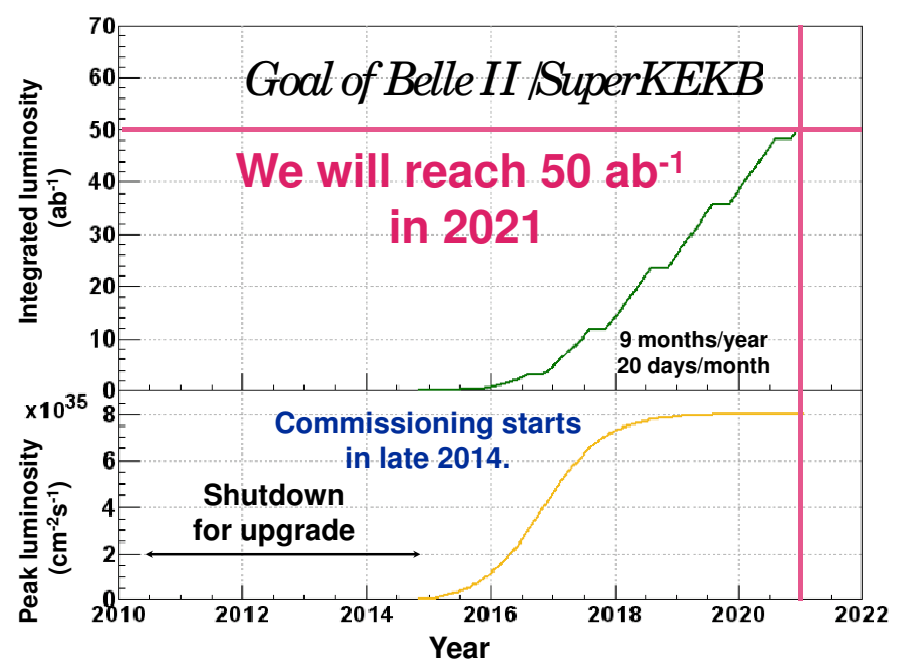

Figure 3: SuperKEKB luminosity projection.

\section{References}

[1] S. Kurokawa and E. Kikutani, Nucl. Instrum. Meth. A499 (2003) 1.

[2] P. Raimondi, Proc. 2nd SuperB Meeting, Frascati (2006).

[3] Y. Suetsugu et al., Nucl. Instrum. Meth. A598 (2009) 372.

[4] M. Kikuchi, Nucl. Instrum. Meth. A556 (2006) 13. 